\title{
Leibniz, Caramuel, Harriot und das Dualsystem
}

Robert Ineichen

Das Zeitalter der Rechenautomaten und der großen Datenverarbeitungsanlagen hat auch das Dualsystem, also das Stellenwertsystem, in welchem die Zahlen allein durch die zwei Ziffern 0 und I dargestellt werden, ziemlich allgemein bekannt werden lassen. Im Zusammenhang mit der Erklärung dieses Dualsystems (dafür auch: Zweiersystem, binäres System, dyadisches System) und der zugehörigen Grundoperationen mit Dualzahlen (Binärzahlen) wird oft Gottfried Wilhelm Leibniz (1646-1716) erwähnt, der sich schon gegen Ende des 17. Jahrhunderts intensiv mit solchen Zahlen beschäftigt hat und im Jahrgang 1703 der Histoire de l'Académie Royale des Sciences eine Arbeit darüber publiziert hat (erschienen in Paris 1705). Manchmal wird Leibniz auch ausdrücklich als „Erfinder“ oder als „Entdecker“ des Dualsystems bezeichnet.

Man kann sich die Fragen stellen, wer als erster über das Dualsystem publiziert hat und welcher Mathematiker sich eigentlich als erster damit beschäftigt hat. Diesen beiden Fragen soll hier etwas nachgegangen werden. - H.J. Zacher, der sich in [I] ebenfalls mit diesen Fragen befasst, geht auch der weiteren Frage nach, was den Anstoß zu solchen Beschäftigungen gegeben haben mag und welche weiteren Einflüsse mitgewirkt haben könnten. Darauf soll hier nicht eingegangen werden.

\section{G. W. Leibniz}

Zu seinen Lebzeiten ist 1705 die oben genannte Arbeit und weiter sind - von ihm angeregt - eine Abhandlung von W.E. Tentzel über die Arithmetica binaria (1705) und eine von P. Dangincourt über die Arithmetica dyadica (I7I0) pu- bliziert worden. Eigentlich sehr wenig, könnte man meinen; es stehen aber diesen wenigen Publikationen zahlreiche weitere gegenüber, die nach dem Tode von Leibniz erschienen sind, und dazu noch in seinem Nachlass eine sehr große Zahl von Abhandlungen, Notizen und Briefen, die sich ebenfalls mit unserem Thema befassen. $^{\prime}$

Seine französisch ${ }^{2}$ geschriebene eben erwähnte Darstellung trägt den Titel Explication de l'Arithmétique binaire. Er schreibt einleitend, dass er sich nur der Ziffern 0 und I bedienen wird und stellt zunächst in einer Tabelle die Zahlen von 0, I, 2, 3 bis 32 im Dualsystem dar, also $0,1,10,11$ bis 100000 . Er weist in dieser Gegenüberstellung auch auf die Perioden hin, die sich in den einzelnen Spalten erkennen lassen, wenn man die aufeinander folgenden Binärzahlen untereinander stellt: Liest man diese Binärzahlen von rechts, so ist wechselweise die erste Ziffer 0 oder I, die zweite zweimal 0 oder zweimal I, die dritte viermal 0 oder viermal I usw.

Er vergisst auch die Praktiker nicht, die beim Wiegen von Massen mit geringem Gewicht mit wenigen Gewichtsstücken auskommen könnten, so ist z. B. ,I3, dargestellt durch II0I, die Summe aus acht, vier und eins“; dies „könnte auch bei den Münzen dienen, um verschiedene Beträge mit wenig Stücken darzustellen“. Es folgen dann Beispiele für die Ausführung der Grundoperationen, analog zu den entsprechenden Rechnungen mit Zahlen im Dezimalsystem, wo jedoch ,nichts auswendig zu lernen ist wie beim gewöhnlich Rechnen", und wo man beim Dividieren „weder zu probieren braucht noch zu erraten“: „,beim Binärsystem wird alles direkt gefunden und bewiesen, wie aus den [...] Beispielen ersichtlich ist". Trotzdem schließt er

I Hans J. Zacher hat die Hauptschriften zur Dyadik von Leibniz publiziert, zusammen mit einer Darstellung der Entstehungsgeschichte der Dyadik bei Leibniz und einem ausführlichen Kommentar [I].

2 Den französischenText und die deutsche Übersetzung findet man z. B. auch in [2]. 
diese Ausführungen mit dem Satz: „Ich empfehle jedoch nicht, dieses Rechensystem an die Stelle der praktischen Dezimalmethode zu setzen." - H.J. Zacher stellt in [I] nach seinen ausführlichen Untersuchungen fest, dass Leibniz ,keine genauen Angaben über den Zeitpunkt der Erfindung seiner Dyadik macht und andererseits die Entdeckung für sich allein beansprucht." Zum Zeitpunkt sind verschiedene Überlegungen gemacht worden; er muss wohl am Anfang der siebziger Jahre liegen. ${ }^{3}$

Bereits in der handschriftlich vorliegenden Abhandlung De Progressione dyadica Pars I (vom I5. März 1679) findet man im Anschluss an die Darstellung einer Multiplikation

1011101
$1011 \frac{1110}{1010}$ (Faktoren 93 und 14, Produkt 1302)
1011101
$\frac{1011101}{10100010110}$

den interessanten Hinweis: ${ }^{4}$

Diese Art Kalkül könnte auch mit einer Maschine - per machinam - ausgeführt werden. [...]. Eine Büchse soll so mit Löchern versehen sein, dass diese geöffnet und geschlossen werden können. Sie sei offen an den Stellen, die jeweils I entsprechen, und bleibe geschlossen an denen, die 0 entsprechen. Durch die offenen Stellen lasse sie kleine Würfel oder Kugeln in Rinnen fallen, durch die anderen nichts. Sie werde so bewegt und von Spalte zu Spalte verschoben, wie die Multiplikation es erfordert. Die Rinnen sollen die Spalten darstellen, und kein Kügelchen soll aus einer Rinne in eine andere gelangen können, es sei denn, nachdem die Maschine in Bewegung gesetzt ist. Dann fließen alle Kügelchen in die nächste Rinne, wobei immer eines weggenommen wird, welches in ein leeres Loch fällt, sofern es allein die Tür passieren will. Denn die Sache kann so eingerichtet werden, dass notwendig immer zwei herauskommen, sonst sollen sie nicht herauskommen.

Bei den letzten zwei Sätzen handelt es sich offenbar um die Beschreibung des „Zweierübergangs": Ein solcher muss stattfinden, wenn zwei oder mehr Kügelchen in einer Rinne sind. Dann müssen zunächst zwei Kügelchen austreten. Von diesen beiden fließt das eine in ,ein leeres Loch" und das andere in die nächste Rinne. Wenn nötig muss dieser Vorgang mehrmals stattfinden. Schließlich befindet sich dann in jeder Rinne nur eine oder überhaupt keine Kugel.

Mit Recht schreibt somit H.J. Greve in [2]: ,Die erste, nach dem binären Prinzip arbeitende Rechenmaschine wurde also bereits von Leibniz im Jahre 1679 skizziert.“

H.J. Zacher [I] stimmt dieser Ansicht zu. Er stellt aber fest, dass sie eigentlich ergänzt werden sollte durch die Aussage: „Das erste, nach dem binären Prinzip arbeitende 〈mechanische) Rechenhilfsmittel wurde bereits von Neper im Jahre 1617 vorgelegt." 5

Leibniz gehört übrigens zusammen mit W. Schickhardt (1592-1635) und Blaise Pascal (1623-1662) auch zu den Wegbereitern der Konstruktion von jenen Rechenmaschinen, die nicht nach dem binären Prinzip arbeiten. - Soviel zum rein Mathematischen. Es soll aber doch noch darauf hingewiesen werden, dass die Dyadik für Leibniz auch ein Bild der biblischen Schöpfung, eine imago creationis, war: Alle Zahlen entstehen aus $I$ und 0 und stellen so gleichsam ,in einem Spiegel die Schöpfung oder den Ursprung der Dinge aus Gott und sonst Nichts" dar. ${ }^{6}$ Dieser interessanten Überlegung von Leibniz, der ja der Philosophie und der Theologie sehr nahe stand, können wir hier nicht nachgehen.

\section{Juan Caramuel y Lobkowitz}

1670 hat der Zisterziensermönch und spätere Bischof J. Caramuel y Lobkowitz seine Mathesis biceps vetus et nova, zwei großformatige, umfangreiche Bände in lateinischer Sprache, publiziert - biceps, also „zweiköpfig“, weil er die damalige ,alte“ und die ,neue“ Mathematik ${ }^{7}$ präsentieren will. Im Band, der der ,alten“ Mathematik gewidmet ist, behandelt er auch die Darstellung der natürlichen Zahlen mit verschiedenen Basen, und unter diesen n-ären Zahlendarstellungen findet man selbstverständlich auch das Zweiersystem. Hier, also 1670, dürfte somit die erste Publikation über das Dualsystem vorliegen.

3 [I, p. $10 \mathrm{ff}$.]

4 Faksimile des lateinischen Textes und deutsche Übersetzung in [2].

5 [I, p. 22]. - Neper (John Napier, 1550-1617) verwendet ebenfalls ein Zweiersystem, das aber hier nicht ein eigentliches Stellenwertsystem ist. Es werden calculi (Rechensteinchen) verwendet, die auf bestimmten Stellen gesetzt werden und so einen zahlenmäßigen Stellenwert erhalten; mit diesen calculi wird bei der Durchführung der Grundoperationen gearbeitet.

6 Aus einem Brief von Leibniz an Herzog Rudolf August vom 8. Mai I696 [I, p. 235]

7 Zur ,neuen“ Mathematik gehört z. B. seine mathematische Behandlung von Würfelspielen, Teilungsproblemen und des Zahlenlottos. Vgl. R. Ineichen [3]. 


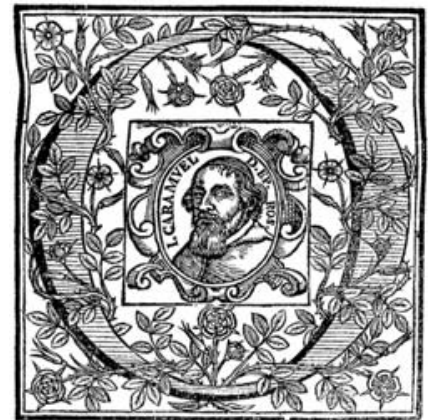

Juan Caramuel y Lobkowitz, 1606-1682
Caramuel (1606-1682), geboren in Madrid, offenbarte schon in früher Jugend großes Talent für Mathematik und Astronomie. Er studierte Philosophie und Theologie und zeigte zeitlebens eine außergewöhnliche Aktivität und Neugierde. Er muss ungeheuer fleißig gearbeitet haben. Dem enzyklopädischen Ideal des Barocks folgend, wollte er in jedes Wissensgebiet eindringen. Seine Publikationen, mehr als siebzig Bände, gelten den verschiedensten Fachgebieten. Allerdings gefiel er sich gelegentlich auch ,in Paradoxen und allem Absonderlichen". ${ }^{8}$ Viele Aufgaben im Dienste der Kirche führten ihn in zahlreiche Länder Europas.

Bei seinen Zahlensystemen handelt es sich immer um Positionssysteme; die Ziffern ersetzt er aber, mit Ausnahme der 0, durch die Buchstaben $a, b, c$, usw. Dies hat den Vorteil, dass er bei Basen, die größer als zehn sind, keine besonderen Benennungen zu erfinden hat. Im ersten Abschnitt, De binaria arithmetica, geht es um das Zweiersystem: Er bezeichnet 0 durch $o$ und 1 durch $a$, dann also $2=2+0=a o$, $3=2+1=a a$, usw., $31=16+8+4+2+1=$ a a a a a und schließlich $32=$ a $о$ oо oo.

Es scheint uns, dass Caramuel mit seinen Ausführungen zeigen wollte, dass ganz verschiedene Basen verwendet werden könnten, wobei sich gewisse Basen durch eine besondere „Natürlichkeit" auszeichnen und deshalb sehr nahe liegend sind, andere uns im Alltag begegnen oder in den Wissenschaften, ohne dass wir sie ausdrücklich als „Basen“ wahrnehmen. Bei der Basis 2 macht er uns auf die Situation bei den Intervallen in der Musik aufmerksam, die er als von der Natur gegeben betrachtet. Hier erzeugt die Verdopplung oder die Halbierung der Saitenlängen jeweils die Oktave.

Es ist hier anzumerken, dass bereits Blaise Pascal in seiner kleinen Abhandlung [4] De numeris multiplicibus (1654 bzw. 1665) festgestellt hat, dass für die Basis 10 unseres üblichen Zahlensystems keine „natürliche Notwendigkeit“, keine necessitas naturae, besteht, sondern dass sie auf Grund einer Konvention, also ex instituto hominum, verwendet wird. 9

Im nächsten Abschnitt, De ternaria arithmetica, arbeitet er mit der Basis 3 und verwendet auBer $o$ und $a$ noch $b$ für die 2; seine Tabelle führt von $o$ bis zu $a a o b=27+9+0+2=38$, aаao $=27+9+3+0=39$, aаaа $=$ $27+9+3+1=40$. Er weist auf die Bedeutung der Zahl 3 in der Theologie hin (die Dreifaltigkeit, das mysterium sanctissimae trinitatis) und auf die Verehrung der Zahl 3 durch die Ägypter, weil (nach Plotin) die Dreizahl „Anfang, Mitte und Ende" - principium, medium et finem hat. Selbstverständlich fehlt hier auch nicht das Vergil-Zitat ,Gott freut sich an der ungeraden Zahl" - Numero Deus impare gaudet - aus der 8. Ekloge (Vers 75). Doch damit nicht genug: Er stellt u. a. fest, dass auch der Himmel ,dreifach“ aufgebaut ist, denn er besteht aus dem Aëreum, dem Aethereum und dem Empyreum, also aus der Luft, dem Äther und dem „Feuerhimmel“ als oberster Weltgegend. Hier und an vielen anderen Stellen seiner Mathesis staunt man immer wieder über seine Phantasie und die vielseitigen Verknüpfungen, die er zu erkennen weiß. Andererseits ist man überrascht, dass Caramuel mit der Beschreibung dieser Zahlensysteme gar keine weiteren mathematischen Überlegungen verbindet oder Hinweise für die Praxis verbindet. ${ }^{10}$

In ähnlicher Art geht es weiter: Es folgen die Basen 4 bis 10, dann noch 12 und 60 . Bei 4 etwa erinnert er nebst vielem anderem an die vier Winde Boreas, Notus (Südwind), Zephyrus (Westwind) und Eurus (Ostwind), dann an die große Rolle, die die "heilige Vierzahl“ bei den Pythagoräern spielt, d.h. an die Tetraktys (die „Vierheit“) der Zahlen I, 2, 3, 4, deren Summe 10 ergibt usw. - Die Basis 5 kann man in den vier Elementen der Antike, Erde, Wasser, Luft und Feuer, ergänzt um die quinta essentia, erkennen. Doch scheint ihm diese Zahl durch die Natur ungenügend fundiert für ein wissenschaftliches System zu sein: non subsistit natura. Nach reichhaltigen weiteren derartigen Betrachtungen gelangt Caramuel zur Sexagenaria Arithmetica, also zur Basis 60, wo er selbstverständlich auf die Grade, Minuten und Sekunden der Astronomen zu sprechen kommt.

\section{Thomas Harriot}

Er ist wohl der erste Erfinder des Dualsystems, wie mehrere Manuskripte in seinem Nachlass zeigen. Er verwendet im Dualsystem die Ziffern 0 und I und zeigt an Beispielen, wie man vom Dezimalsystem ins Dualsystem und umgekehrt übergehen kann (Conversio bzw. Reductio). An weiteren Beispielen demonstriert er die Grundoperationen. "I

8 Mehr darüber bei R. Ineichen [3].

9 Nach M. Cantor [5, p. 783] war Pascal in dieser Sache „Vorgänger, aber jedenfalls unbekannter Vorgänger von Caramuel.".

${ }^{10}$ In etlichen anderen Kapiteln seiner Mathesis biceps geht er hingegen oft auch seinen mathematischen Interessen nach, so z. B. bei der Behandlung der Teilbarkeit von natürlichen Zahlen, bei den Würfelspielen, beim Zahlenlotto, bei geometrischen Konstruktionen.

I" John W. Shirley hat als erster 1950 darauf hingewiesen und darüber 195 I publiziert [6]. 
Thomas Harriot (1560-162I) war Mathematiker, Physiker und Astronom. Er trat zunächst in die Dienste von Sir Walter Raleigh und wurde von diesem zur Vermessung von Virginia (North Carolina) ausgesandt; später lebte er zunächst in Irland, dann in England. - Die meisten seiner Untersuchungen sind nur aus seinem handschriftlichen Nachlass bekannt; die Artis analyticae praxis, die bloß elementare Mathematik enthält, liegt gedruckt vor.

Shirley weist darauf hin, dass Harriot keineswegs nur mit dem Dualsystem arbeitet, sondern auch in Zahlensystemen mit den Basen 3, 4, 5 usw. Er betont, wie die vorliegenden Akten vor allem auch ,the clarity with which he saw the problems and the facility with which he used the techniques of fundamental calculation in the nondecimal systems" zeigen.

Doch Harriot sah keine praktischen Anwendungen für seine verschiedenen neuen Zahlensysteme, und so verfolgte er diese Untersuchungen nicht weiter. Shirley schließt seine Ausführungen mit einem Satz, mit dem wohl die meisten Mathematiker sehr gerne einverstanden sind; mit ihm wollen wir hier abbrechen:

If there is a lesson in this tale, it is that useless knowledge may someday be useful - theory, impractical though it seem, may well be of real and practical value in situations as yet unimagined.

\section{Literatur}

[I] H. J. Zacher (1973): Die Hauptschriften zur Dyadik von G.W. Leibniz - Ein Beitrag zur Geschichte des binären Zahlensystems. Frankfurt a. M.: Vittorio Klostermann.

[2] E. Hochstetter e.a. (1966): Herrn von Leibniz' Rechnung mit Null und Eins. Berlin: Siemens AG.

[3] R. Ineichen (1999) Juan Caramuels Behandlung der Würfelspiele und des Zahlenlottos. N.T.M - Internationale Zeitschrift für Geschichte und Ethik der Naturwissenschaften, Technik und Medizin 7 (2I30).

R. Ineichen (1999) Über die Kybeia und die Arithmomantica von Juan Caramuel y Lobkowitz - ein Kapitel aus der Frühgeschichte der Wahrscheinlichkeitsrechnung. Bull. Soc. Frib. Sc. Nat. 87 (5-55).

[4] B. Pascal (1954): Euvres complètes. Texte établi, présenté et annoté par. J. Chevalier (p. 159-165). Paris: Gallimard.

[5] M. Cantor (19/3): Geschichte der Mathematik, Bd. 2. Leipzig: Teubner.

[6] J. W. Shirley (195I): Binary Numeration before Leibniz. American Journal of Physics 19 (452-454).

\section{Bildnachweise}

S. 13: Helmholtz-Zentrum für Kulturtechnik der Humboldt-Universität zu Berlin, http://www. sammlungen.hu-berlin.de/dokumente/283/ S. 14: Caramuel electronicus - Opera et studia electronica Joannis Caramuel Lobkowitz cura et studio Jacobi Schmutzii, Doctoris Sorbonici, http:// pagesperso-orange.fr/caramuel/index.html

S. 15: Thomas Harriot Seminar, http://www.rensoc.org. uk/ths/index.htm

\section{Adresse des Autors}

Prof. émérite Dr. Robert Ineichen

Institut de mathématiques

Université de Fribourg

1700 Fribourg

Schweiz

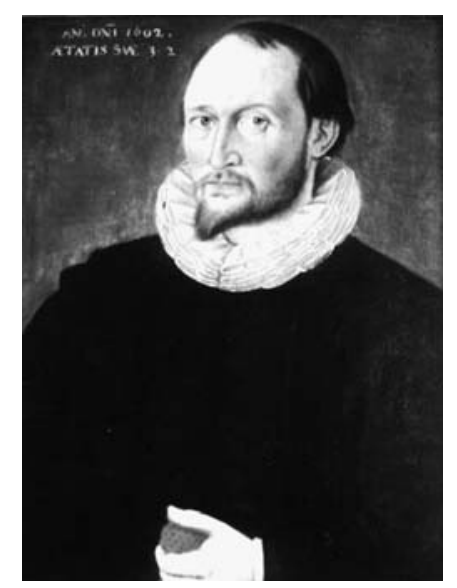

Thomas Harriot, |560-|62| 